PROCEEDINGS OF THE

AMERICAN MATHEMATICAL SOCIETY

Volume 125, Number 6, June 1997, Pages 1705-1709

S $0002-9939(97) 03850-1$

\title{
APPROXIMATION OF FIXED POINTS OF STRONGLY PSEUDOCONTRACTIVE MAPS WITHOUT LIPSCHITZ ASSUMPTION
}

\author{
ZHOU HAIYUN AND JIA YUTING
}

(Communicated by Palle E. T. Jorgensen)

\begin{abstract}
In the present paper, the following result is shown: Let $X$ be a real Banach space with a uniformly convex dual $X^{*}$, and let $K$ be a nonempty closed convex and bounded subset of $X$. Assume that $T: K \rightarrow K$ is a continuous strong pseudocontraction. Let $\left\{\alpha_{n}\right\}_{n=1}^{\infty}$ and $\left\{\beta_{n}\right\}_{n=1}^{\infty}$ be two real sequences satisfying
\end{abstract}

(i) $0<\alpha_{n}, \beta_{n}<1$ for all $n \geq 1$;

(ii) $\sum_{n=1}^{\infty} \alpha_{n}=\infty$; and

(iii) $\alpha_{n} \rightarrow 0, \beta_{n} \rightarrow 0$ as $n \rightarrow \infty$.

Then the Ishikawa iterative sequence $\left\{x_{n}\right\}_{n=1}^{\infty}$ generated by

$$
\text { (I) }\left\{\begin{array}{l}
x_{1} \in K, \\
x_{n+1}=\left(1-\alpha_{n}\right) x_{n}+\alpha_{n} T y_{n}, \\
y_{n}=\left(1-\beta_{n}\right) x_{n}+\beta_{n} T x_{n}, n \geq 1,
\end{array}\right.
$$

converges strongly to the unique fixed point of $T$.

\section{INTRODUCTION AND PRELIMINARIES}

Let $X$ be a normed linear space, $K \subseteq X$. A mapping $T: K \rightarrow K$ is said to be strongly pseudocontractive if there exists $t>1$ such that the inequality

$$
\|x-y\| \leq\|(1+r)(x-y)-r t(T x-T y)\|
$$

holds for every $x, y \in K$ and $r>0$. A mapping $U$ with domain $D(U)$ and range $R(U)$ in $X$ is called accretive if the following inequality

$$
\|x-y\| \leq\|x-y+s(U x-U y)\|
$$

holds for every $x, y \in D(U)$ and for all $s \geq 0$. Browder [1, Prop. 1] proved that $T$ is pseudocontractive if and only if $(I-T)$ is accretive, where $I$ denotes the identity operator.

Let $X$ be a real Banach space with a dual $X^{*}$. The normalized duality mapping $J: X \rightarrow 2^{X^{*}}$ is defined by

$$
J(x)=\left\{f \in X^{*} ;\langle x, f\rangle=\|x\|^{2},\|x\|=\|f\|\right\},
$$

where $\langle\cdot, \cdot\rangle$ denotes the generalized duality pairing. It is well known that if $X^{*}$ is strictly convex, then $J$ is single valued and such that $J(-x)=-J(x), J(t x)=$

Received by the editors December 5, 1995.

1991 Mathematics Subject Classification. Primary 47H17.

Key words and phrases. The Ishikawa iteration, strong pseudocontraction, strictly convex Banach space. 
$t J(x)$ for all $t \geq 0, x \in X$. If $X^{*}$ is uniformly convex, then $J$ is uniformly continuous on the bounded sets of $X$ (see, e.g., Barbu [2])

By means of the normalized duality mapping $J$, we can give the following equivalent definition for accretive mapping.

A mapping $A: K \rightarrow K$ is said to be accretive if for each $x, y$ in $K$ there exists $j \in J(x-y)$ such that

$$
\langle A x-A y, j\rangle \geq 0 \text {. }
$$

A mapping $A: K \rightarrow K$ is called strongly accretive if for each $x, y \in K$ there exists $j \in J(x-y)$ such that

$$
\langle A x-A y, j\rangle \geq k\|x-y\|^{2}
$$

for some constant $k>0$. Without loss of generality we shall assume that $k \in(0,1)$.

In [3], Bogin proved that $U$ is strongly pseudocontractive if and only if $I-U$ is strongly accretive.

Very recently, Chidume [4] proved the following result: If $X$ is a real Banach space with a uniformly convex dual $X^{*}$, and $K$ is a nonempty closed convex and bounded subset of $X$, and $T: K \rightarrow K$ is a Lipschitz strongly pseudocontractive mapping, the Ishikawa iterative sequence $\left\{x_{n}\right\}_{n=1}^{\infty}$ generated by (I) converges strongly to the unique fixed point of $T$. However, when $T$ is continuous strongly pseudocontractive, it is still an open question whether or not the Ishikawa iterative sequence $\left\{x_{n}\right\}_{n=1}^{\infty}$ defined by (I) converges strongly to a fixed point of $T$.

It is our purpose in this paper to solve the above question by proving the following much more general result: If $X$ is a real Banach space with a uniformly convex dual $X^{*}, K$ is a nonempty bounded closed convex subset of $X$, and $T: K \rightarrow K$ is a continuous strongly pseudocontractive mapping, then the Ishikawa iteration sequence $\left\{x_{n}\right\}_{n=1}^{\infty}$ defined by (I) converges strongly to the unique fixed point of $T$.

We need the following lemmas.

Lemma 1.1. Let $X^{*}$ be strictly convex; then

$$
\|x+y\|^{2} \leq\|x\|^{2}+2\langle y, J(x+y)\rangle
$$

for all $x, y \in X$.

Proof. For arbitrary fixed $x, y \in X$, let $\varphi(s)=\|x+s y\|^{2}, s \in[0,+\infty)$; then $\varphi:[0,+\infty) \rightarrow[0, \infty)$ is a convex function.

To see this, for every $s_{1}, s_{2} \in[0,+\infty), t \in[0,1]$, we have

$$
\begin{aligned}
\varphi\left(t s_{1}\right. & \left.+(1-t) s_{2}\right)=\left\|t\left(x+s_{1} y\right)+(1-t)\left(x+s_{2} y\right)\right\|^{2} \\
& \leq t^{2}\left\|x+s_{1} y\right\|^{2}+(1-t)^{2}\left\|x+s_{2} y\right\|^{2}+2 t(1-t)\left\|x+s_{1} y\right\|\left\|x+s_{2} y\right\| \\
& \leq\left(t^{2}+t(1-t)\right)\left\|x+s_{1} y\right\|^{2}+\left((1-t)^{2}+t(1-t)\right)\left\|x+s_{2} y\right\|^{2} \\
& =t \varphi\left(s_{1}\right)+(1-t) \varphi\left(s_{2}\right) .
\end{aligned}
$$

Since $\varphi:[0,+\infty) \rightarrow[0,+\infty)$ is a convex function, as $s>t>0$, we have

$$
\begin{aligned}
\varphi(t) & =\varphi\left(\frac{t}{s} \cdot s+\left(1-\frac{t}{s}\right) \cdot 0\right) \\
& \leq \frac{t}{s} \varphi(s)+\left(1-\frac{t}{s}\right) \varphi(0) \\
& =\frac{t}{s}(\varphi(s)-\varphi(0))+\varphi(0)
\end{aligned}
$$


and then

$$
t^{-1}(\varphi(t)-\varphi(0)) \leq s^{-1}(\varphi(s)-\varphi(0)) .
$$

Hence, function $t \mapsto t^{-1}(\varphi(t)-\varphi(0))$ is increasing. As a result, $\lim _{t \rightarrow 0^{+}} t^{-1}(\varphi(t)-\varphi(0))$ exists and

$$
\|x+y\|^{2}-\|x\|^{2} \geq \lim _{t \rightarrow 0^{+}} t^{-1}(\varphi(t)-\varphi(0))=\left\langle y, \operatorname{grad}\|x\|^{2}\right\rangle=2\langle y, J(x)\rangle,
$$

so that, for all $x, y \in X$ we have

$$
\|x+y\|^{2} \geq\|x\|^{2}+2\langle y, J(x)\rangle .
$$

Now by replacing $x$ by $x-y$ in (6) we get

$$
\|x\|^{2} \geq\|x-y\|^{2}+2\langle y, J(x-y)\rangle .
$$

Replacing $x$ by $-x$ in (7), we obtain

$$
\|x\|^{2} \geq\|x+y\|^{2}-2\langle y, J(x+y)\rangle .
$$

Thus, we have

$$
\|x+y\|^{2} \leq\|x\|^{2}+2\langle y, J(x+y)\rangle
$$

for all $x, y \in X$.

Lemma 1.2. Let $\left\{\rho_{n}\right\}_{n=1}^{\infty}$ be a nonnegative real sequence satisfying

$$
\rho_{n+1} \leq\left(1-\lambda_{n}\right) \rho_{n}+\sigma_{n}
$$

where $\lambda_{n} \in[0,1], \sum_{n=1}^{\infty} \lambda_{n}=\infty$, and $\sigma_{n}=o\left(\lambda_{n}\right)$. Then $\rho_{n} \rightarrow 0$ as $n \rightarrow \infty$.

Proof. Let $\alpha=\inf \left\{\rho_{n}: n \geq 1\right\}$; then $\alpha=0$. If not, assume that $\alpha>0$; then for all $n \geq 1, \rho_{n} \geq \alpha>0$.

Since $\sigma_{n}=o\left(\lambda_{n}\right)$ we know that

$$
\sigma_{n}<\frac{1}{2} \rho_{n} \lambda_{n}
$$

for all large $n$.

From (9), we get

$$
\rho_{n+1}<\left(1-\lambda_{n}\right) \rho_{n}+\frac{1}{2} \rho_{n} \lambda_{n}=\left(1-\frac{1}{2} \lambda_{n}\right) \rho_{n} .
$$

By a simple iteration we get that

$$
0 \leq \rho_{n+1} \leq \exp \left\{-\frac{1}{2} \sum_{j=1}^{n} \lambda_{j}\right\} \rho_{1} \rightarrow 0 \text { as } n \rightarrow \infty .
$$

i.e., a contradiction. Thus there exists some subsequence of $\left\{\rho_{n}\right\}$, say $\left\{\rho_{n_{j}}\right\}$, such that $\rho_{n_{j}} \rightarrow 0$ as $j \rightarrow \infty$. Hence for arbitrary given $\epsilon>0$, there exists large integer $j_{0} \geq 1$ such that

$$
\sigma_{n}<\lambda_{n} \epsilon, \rho_{n_{j_{0}}}<\epsilon
$$

for all large $n \geq n_{j_{0}}$.

By virtue of (9) we have

$$
\rho_{n_{j_{0}+1}} \leq\left(1-\lambda_{n_{j_{0}}}\right) \epsilon+\lambda_{n_{j_{0}}} \epsilon=\epsilon
$$

by induction, we have

$$
\rho_{n_{j_{0}}+k} \leq \epsilon, \text { for all } k \geq 1 .
$$

This shows $\rho_{n} \rightarrow 0$ as $n \rightarrow \infty$. 


\section{MAIN RESUlts}

Now we prove the main results.

Theorem 2.1. Let $X$ be a real Banach space with a uniformly convex dual $X^{*}, K$ be a nonempty closed convex bounded subset of $X$, and $T: K \rightarrow K$ be a continuous strongly pseudocontractive mapping. Then the Ishikawa iterative sequence $\left\{x_{n}\right\}_{n=1}^{\infty}$ defined by (I) converges strongly to the unique fixed point of $T$.

Proof. The existence of a fixed point follows from Deimling [5]. Let $q$ be a fixed point of $T$. Since $T: K \rightarrow K$ is strongly pseudocontractive, $I-T$ is strongly accretive, and for every $x, y \in K$

$$
\langle(I-T) x-(I-T) y, J(x-y)\rangle \geq k\|x-y\|^{2},
$$

where $k=(t-1) t^{-1}$ (see, e.g., [3]).

From (10) we obtain

$$
\langle T x-T y, J(x-y)\rangle \leq(1-k)\|x-y\|^{2}
$$

for all $x, y \in K$.

Using (5), (11) and (I), we get

$$
\begin{aligned}
\| x_{n+1} & -q\left\|^{2}=\right\|\left(1-\alpha_{n}\right)\left(x_{n}-q\right)+\alpha_{n}\left(T y_{n}-T q\right) \|^{2} \\
\leq & \left(1-\alpha_{n}\right)^{2}\left\|x_{n}-q\right\|^{2}+2 \alpha_{n}\left\langle T y_{n}-T q, J\left(x_{n+1}-q\right)\right\rangle \\
= & \left(1-\alpha_{n}\right)^{2}\left\|x_{n}-q\right\|^{2}+2 \alpha_{n}\left\langle T y_{n}-T q, J\left(x_{n+1}-q\right)-J\left(y_{n}-q\right)\right\rangle \\
& +2 \alpha_{n}\left\langle T y_{n}-T q, J\left(y_{n}-q\right)\right\rangle \\
\leq & \left(1-\alpha_{n}\right)^{2}\left\|x_{n}-q\right\|^{2}+2 \alpha_{n} a_{n}+2 \alpha_{n}(1-k)\left\|y_{n}-q\right\|^{2}
\end{aligned}
$$

where

$$
a_{n}=\left\langle T y_{n}-T q, J\left(x_{n+1}-q\right)-J\left(y_{n}-q\right)\right\rangle .
$$

Now we shall show $a_{n} \rightarrow 0$ as $n \rightarrow \infty$. Observe that $\left\{T y_{n}-T q\right\}$ is bounded; then to show $a_{n} \rightarrow 0$ as $n \rightarrow \infty$, it suffices to show

$$
J\left(x_{n+1}-q\right)-J\left(y_{n}-q\right) \rightarrow 0 \text { as } n \rightarrow \infty .
$$

Indeed, since $X^{*}$ is uniformly convex, $J$ is uniformly continuous on any bounded subset of $X$. Noting that $\left(x_{n+1}-q\right)-\left(y_{n}-q\right)=x_{n+1}-y_{n}=\left(\beta_{n}-\alpha_{n}\right) x_{n}+$ $\alpha_{n} T y_{n}-\beta_{n} \rightarrow 0$ as $n \rightarrow \infty$, we see that

$$
J\left(x_{n+1}-q\right)-J\left(y_{n}-q\right) \rightarrow 0 \text { as } n \rightarrow \infty .
$$

Again using (5) and (I), we have

$$
\begin{aligned}
\left\|y_{n}-q\right\|^{2} & =\left\|\left(1-\beta_{n}\right)\left(x_{n}-q\right)+\beta_{n}\left(T x_{n}-T q\right)\right\|^{2} \\
& \leq\left(1-\beta_{n}\right)^{2}\left\|x_{n}-q\right\|^{2}+2 \beta_{n}\left\langle T x_{n}-T q, J\left(y_{n}-q\right)\right\rangle \\
& \leq\left\|x_{n}-q\right\|^{2}+M_{1} \beta_{n}
\end{aligned}
$$

for some constant $M_{1}>0$, since $\left\langle T x_{n}-T q, J\left(y_{n}-q\right)\right\rangle$ is bounded. 
Substituting (13) in (12) yields

$$
\begin{aligned}
\left\|x_{n+1}-q\right\|^{2} & \leq\left(\left(1-\alpha_{n}\right)^{2}+2(1-k) \alpha_{n}\right)\left\|x_{n}-q\right\|^{2} \\
& +2 \alpha_{n}\left(a_{n}+(1-k) M_{1} \beta_{n}\right) \\
& =\left(1-2 \alpha_{n}+\alpha_{n}^{2}+2 \alpha_{n}-2 k \alpha_{n}\right)\left\|x_{n}-q\right\|^{2} \\
& +2 \alpha_{n}\left(a_{n}+(1-k) M_{1} \beta_{n}\right) \\
& =\left(1+\alpha_{n}^{2}-2 k \alpha_{n}\right)\left\|x_{n}-q\right\|^{2}+\sigma_{n},
\end{aligned}
$$

where $\sigma_{n}=2 \alpha_{n}\left(a_{n}+(1-k) M_{1} \beta_{n}\right)$.

We choose a large positive integer $N$ such that for all $n>N$

$$
\alpha_{n}<k \text {. }
$$

Thus, the above inequality yields

$$
\left\|x_{n+1}-q\right\|^{2} \leq\left(1-k \alpha_{n}\right)\left\|x_{n}-q\right\|^{2}+\sigma_{n} .
$$

Set $\rho_{n}=\left\|x_{n}-q\right\|^{2}$ and $\lambda_{n}=k \alpha_{n}$. Then

$$
\rho_{n+1} \leq\left(1-\lambda_{n}\right) \rho_{n}+\sigma_{n},
$$

with $\lambda_{n} \in[0,1], \sum_{n=1}^{\infty} \lambda_{n}=\infty$ and $\sigma_{n}=o\left(\lambda_{n}\right)$. By Lemma 1.2 we see that $\rho_{n} \rightarrow 0$ as $n \rightarrow \infty$.

Now we prove the uniqueness. Suppose $q_{1}$ is another fixed point of $T$; then by (10) we have

$$
0=\left\langle(q-T q)-\left(q_{1}-T q_{1}\right), J\left(q-q_{1}\right)\right\rangle \geq k\left\|q-q_{1}\right\|^{2},
$$

which implies $q_{1}=q$.

The proof of this theorem is completed.

Remark. Our theorem extends Theorem 2 of Chidume [4] to a larger class of continuous strongly pseudocontractive mappings; thus we have solved an open question put forth by Chidume in [4].

\section{REFERENCES}

[1] F. E. Browder, Nonlinear operators and nonlinear equation of evolution in Banach spaces, Proc. Sympos. Pure. Math., 18(1976). MR 53:8982

[2] V. Barbu, Nonlinear Semigroups and Differential Equations in Banach Spaces, Noordhoff, Leyden, 1976. MR 52:11666

[3] J. Bogin, On strict pseudo-contractions and a fixed point theorem, Technion Preprint MT-29, Haifa, 1974.

[4] C. E. Chidume, Approximation of fixed points of strongly pseudocontractive mappings, Proc. Amer. Math. Soc. 120, No.2 (1994), 545-551. MR 94d:47056

[5] K. DEIMLING, Zeros of accretive operators, Manucripta Math. 13 (1974), 283-288. MR 50:3030

Department of Mathematics, Shijiazhuang Engineering College, Shijiazhuang 050003, People's Republic of China

Department of Mathematics, Hebei Teachers University, Shijiazhuang 050016, PeoPLE'S Republic OF ChinA 